\title{
IdeAs
}

Idées d'Amériques

$6 \mid 2015$

Migrer dans les Amériques

\section{Une conséquence imprévue de la loi sur l'immigration de 1965 : la mise en illégalité des migrants mexicains et la montée de la «menace latino »}

James Cohen

\section{CpenEdition}

\section{Journals}

Édition électronique

URL : https://journals.openedition.org/ideas/1292

DOI : 10.4000/ideas. 1292

ISSN : 1950-5701

Éditeur

Institut des Amériques

Référence électronique

James Cohen, « Une conséquence imprévue de la loi sur l'immigration de 1965 : la mise en illégalité des migrants mexicains et la montée de la « menace latino » », IdeAs [En ligne], 6 | 2015, mis en ligne le 17 décembre 2015, consulté le 19 octobre 2022. URL : http://journals.openedition.org/ideas/1292 ; DOI : https://doi.org/10.4000/ideas.1292

Ce document a été généré automatiquement le 19 octobre 2022.

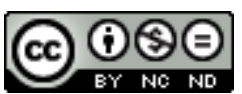

Creative Commons - Attribution - Pas d'Utilisation Commerciale - Pas de Modification 4.0 International - CC BY-NC-ND 4.0

https://creativecommons.org/licenses/by-nc-nd/4.0/ 


\title{
Une conséquence imprévue de la loi sur l'immigration de 1965 : la mise en illégalité des migrants mexicains et la montée de la « menace latino »
}

\author{
James Cohen
}

1 Il est frappant de voir à quel point la loi sur l'immigration de 1965 est sujette à des interprétations différentes voire contradictoires. La plupart des historiens, suivant l'administration Johnson et les législateurs qui ont promu le texte, la définissent comme la mesure par laquelle le principe de non-discrimination ethnique, raciale et nationale a été introduite dans la loi sur l'immigration, par transposition, à l'époque du Civil Rights Act (1964) et du Voting Rights Act (1965). Autrement dit, c'est une loi qui introduit enfin de l'équité dans un domaine longtemps régi par des systèmes de quotas discriminatoires voire racistes (voir l'article de B. Deschamps dans ce dossier). À partir de là, de nombreux historiens, sociologues et politistes soulignent, chiffres à l'appui, à quel point la loi a contribué à la diversification des flux et, partant, à une véritable mondialisation par l'immigration de la société états-unienne - même si le contenu des flux à venir était loin d'être prévu à l'époque (Tichenor D., 2002 : 209-218 ; Ngai M., 2004 : 258-264 ; Zolberg A., 2006 : 333-336).

Quand on entre en détail dans la teneur des débats au Congrès sur le texte, on s'aperçoit que ses principaux effets n'étaient pas prévus à l'époque. Loin de mettre l'accent sur les flux migratoires renouvelés ou sur la "diversité » qu'ils allaient apporter, le sénateur Edward Kennedy a promis à ses collègues réticents que «nos villes ne seront pas inondées par un million d'immigrés chaque année... La composition ethnique de ce pays ne sera pas affectée " (Ong Hing B., 2003: 95). Les législateurs s'attendaient à une augmentation modeste des flux en provenance de l'Europe de l'Est et du Sud mais ne prévoyaient pas d'immigration nouvelle d'Asie ou d'Afrique. Quant à la forte poussée de l'immigration latino-américaine, selon Douglas Massey et Karen 
Pren (2012: 1), elle s'est produite « en dépit du nouveau système plutôt qu'à cause de celui-ci ».

Des analyses plus approfondies mettent en évidence une grandie ironie, à savoir qu'en imposant aux migrants des Amériques (the Western Hemisphere), à partir de 1968, un quota régional de 120000 entrées qui n'avait pas existé jusqu'alors, la loi, accompagnée de ses amendements des années 1970, a eu également pour effet de rendre beaucoup plus difficile l'immigration par la voie légale de ceux qui étaient sociologiquement les plus nombreux, à savoir les Mexicains. En effet, l'amendement de 1976 a limité à 20000 le nombre de visas annuels attribués à chaque pays de l'hémisphère, indépendamment de sa taille ou de sa relation migratoire établie avec les États-Unis. Autrement dit, cette loi tant vantée pour ses vertus antiracistes a eu aussi pour effet de contribuer à la mise en illégalité de la principale composante de la minorité latino-américaine émergente. C'est le sens d'une analyse que les sociologues Douglas Massey et Jorge Durand et leurs collaborateurs mettent en forme et affinent progressivement dans une série de livres et d'articles publiés depuis 1999.

Il importe de noter qu'au moment où la loi a été votée, l'État fédéral venait de mettre fin au programme Bracero (1942-1964), lequel, à son apogée entre les années 1956 et 1958, envoyait plus de 400000 travailleurs agricoles mexicains annuellement aux ÉtatsUnis (données de l'INS citées par Tichenor D., 2002 : 210). L'abolition du programme a été justifiée comme un acte de suppression d'un système de gestion de la main-d'œuvre étrangère qui se prêtait à beaucoup d'exploitation abusive des salariés. Les travailleurs saisonniers que ce programme fournissait aux entreprises agricoles étaient souvent mal payés, ou privés de salaire, et obligés de vivre dans de conditions insalubres. Des syndicats et des groupes religieux s'opposaient au programme au nom des intérêts des migrants eux-mêmes et des travailleurs américains (Calavita K. 1992; Massey D., Durand J. et Malone N., 2002: 35-41; Tichenor D., 2002: 209-211). Son non renouvellement, conjugué à l'imposition de limites au nombre de visas accordés aux Mexicains, a réduit de façon drastique les possibilités d'immigration légale depuis le Mexique.

5 À partir de l'adoption de la loi, « la migration irrégulière (undocumented) en est venue à dominer les flux migratoires vers les États-Unis ». De 1965 à 1986, environ 28 millions de Mexicains sont entrés aux Etats-Unis en tant qu'immigrés « sans-papiers », contre seulement un 1,3 millions d'immigrés légaux et 46000 travailleurs contractuels » (Massey D. et. al., 2002 : 45). Ces migrations étaient dans une grande mesure circulaires, puisqu'il était relativement facile à l'époque pour un migrant en âge de travailler de traverser la frontière plusieurs fois. Ces flux ont pu maintenir leur caractère globalement circulaire pour un temps encore. En effet, aux 28 millions d'entrées en ces années correspondaient 23,4 millions de départs, pour une augmentation nette de 4,6 millions. Mais plus tard, avec le renforcement progressif des dispositifs militarisés de sécurité à la frontière, les immigrés en situation irrégulière, une fois installés aux ÉtatsUnis, se sont trouvés empêchés de quitter le territoire, de peur de ne plus pouvoir y retourner s'ils repartaient au Mexique.

6 Le décalage entre les objectifs proclamés de la loi et ses effets réels ne s'explique pas, pour Massey et ses co-auteurs, par une animosité particulière envers les Mexicains ou autres Latino-Américains. Le problème fondamental à leurs yeux est la tendance de l'État fédéral à formuler des lois sur l'immigration qui font abstraction des réalités sociologiques du terrain. La loi de 1965 a été adoptée, écrit Massey, « sans comprendre 
clairement comment et pourquoi les gens émigrent vers les États-Unis depuis tel ou tel pays d'origine ou comment l'action du Congrès affecte ces logiques » (Massey D., 2015). Cependant, l'augmentation des flux de migrants en situation irrégulière pendant cette période a permis à des activistes politiques (certains membres de la police des frontières [U.S. Border Patrol], des élus conservateurs, des militants anti-immigrés) de "définir l'immigration latino comme une menace grave pour la nation ", contribuant ainsi à la montée de ce que Leo R. Chavez appelle "le récit de la menace latino " (Chavez L., 2008 ; Massey D. et Pren K., 2012 : 4).

7 Cette dynamique s'est prolongée et renforcée par une série de mesures, ponctuée par des mobilisations politiques pour la défense de la sécurité frontalière, la souveraineté nationale, voire, dans certains contextes, pour la défense d'une conception particulière de l'identité états-unienne (Reimer D., 1998 ; Cohen J., 2012). La loi de 1986 (Immigration Reform and Control Act - IRCA), résultat d'un compromis et d'un marchandage politique, a certes permis à trois millions d'immigrés en situation irrégulière d'être régularisés et placés sur une voie conduisant à la naturalisation, mais elle a imposé aussi - en tout cas sur le papier - des inspections et des sanctions contre des employeurs qui utilisaient une main-d'œuvre irrégulière. Comme plusieurs autres mesures adoptées depuis, IRCA renforce les effectifs et augmente le budget de la Border Patrol. Après 1986, écrivent Massey et. al., "le contrôle de la frontière se ritualise en tant que figure imposée (mandatory public performance)", faisant l'objet d'une concurrence politique et de beaucoup de « gestes symboliques» $(2002: 89)$.

Quelques années plus tard, des élus et des officiers de la Border Patrol politiquement motivés organisent des manœuvres à la frontière visant à donner un tour dramatique à l'immigration irrégulière: Operation Hold the Line (El Paso, septembre 1993), puis Operation Gatekeeper (San Diego, septembre 1994). Les élections californiennes de 1994 sont célébrées sous le signe de la «Proposition 187 », initiative référendaire invitant les électeurs à interdire certains types de services sociaux aux immigrés en situation irrégulière. À la même époque, la construction de barrières frontalières à San Diego et à El Paso signale le début d'un processus de militarisation de la frontière qui se traduira par l'implantation d'un matériel de surveillance sophistiqué et par plusieurs augumentations successives des effectifs de la Border Patrol (Nevins J., 2002 ; Andreas, P., 2009). Entre 2000 et 2008 le nombre d'agents est passé de 4000 à plus de 18000 (Hernández K., 2010 : 232). Fin 2015 l'agence en compte plus de 21000.

9 La loi Illegal Immigration Reform and Immigrant Repsonsibility Act (IIRIRA) signée par William Clinton en 1996 a renforcé par une panopolie de mesures les dispositifs destinés à dissuader les migrants de traverser la frontière et à réduire leurs droits une fois installés sur le territoire : construction d'une triple barrière à San Diego, imposition de sanctions plus sévères pour les passeurs et les sans-papiers, implantation de nouvelles technologies de détection de mouvements, imposition de limites aux formes d'assistance sociale que les sans-papiers pouvaient recevoir etc. (Massey D. et. al., 2002: 95). La même année, la loi Personal Responsiblity and Work Opportunity Reconciliation Act interdit non seulement aux sans-papiers (reprenant au passage les mesures jugées anti-constitutionnelles lorsque l'État de Californie les avaient adoptées par référendum), mais aussi aux immigrés autorisés l'accès aux programmes fédéraux d'assistance.

Les attentats du 11 septembre 2001, qui mettent une fin abrupte aux négociations en cours entre le Mexique et les États-Unis à propos d'un possible compromis historique 
bilatéral à propos des immigrés irréguliers (Castañeda J., 2007), impriment leur marque sécuritaire à toute la période, d'où la création en 2002 du Department of Homeland Security et la redéfinition de l'immigration et des affaires frontalières comme questions relevant directement de la sécurité nationale. D'où, aussi, l'échec de toutes les tentatives depuis lors $(2006,2007,2013$ etc.) d'obtenir par la voie législative, la " réforme intégrale de l'immigration » souhaitée par Bush puis par Obama. C'est ainsi que les undocumented, au nombre de 10 ou 11 millions en 2015, dont environ 5,6 millions de Mexicains (Krogstad J. et Passel J., 2015), se transforment en une caste marginalisée de non-citoyens largement privés de droits (Bosniak L., 2006), sauf dans la mesure où les mesures prises par l'administration Obama depuis 2012 sur la base de sa seule autorité exécutive ont réussi, par le programme Deferred Action for Childhood Arrivals (DACA) à régulariser provisoirement une catégorie des sans-papiers : les jeunes arrivés aux États-Unis en bas âge avec leurs parents.

11 L'immigration mexicaine aux États-Unis a atteint un pic en 2007 avec 12,8 millions de ressortissants, dont la majorité (6,9 millions ou 53 \%) était en situation irrégulière. Bien qu'il y ait, depuis 2012, plus de retours vers le Mexique que d'arrivées, le Mexique demeure la principale source d'étrangers aux États-Unis (28\% en 2013), ainsi que la principale source d'immigrés en situation irrégulière (González-Barrera A., 2015).

12 Si la loi de 1965 est loin d'être directement responsable de tous les dysfonctionnements du système migratoire états-unien aujourd'hui, elle apparaît néanmoins comme la première impulsion forte donnée à la dynamique de mise en illégalité des migrants mexicains. Cette question est devenue l'un des principaux vecteurs d'une forte polarisation qui divise le champ politique aux États-Unis. La « diversité » que la loi a rendue possible n'est pas un acquis définitif, mais reste un enjeu, comme le montre tout particulièrement le cas des Mexicains.

\section{BIBLIOGRAPHIE}

Andreas, Peter, Border Games: Policing the U.S.-Mexico Divide, Cornell University Press, 2009 (2e éd.).

Bosniak, Linda, The Citizen and the Alien. Dilemmas of Contemporary Membership, Princeton

University Press, 2006.

Calavita, Kitty, Inside the State: The Bracero Program, Immigration and the INS, Routledge, 1992.

Castañeda, Jorge G., Ex Mex: From Migrants to Immigrants, New Press, 2007.

Chavez, Leo R., The Latino Threat: Constructing immigrants Citizens and the Nation, Stanford University Press, 2008.

Cohen, James, A la poursuite des « illégaux ». Politiques et mouvements anti-immigrés aux Etats-Unis, éd. Le Croquant (coll. Terra), 2012.

Fitzgerald, David S. et David Cook-Martin, "The Geopolitical Origins of the U.S. Immigration Act of 1965”, Migration Policy Institute, Migration Information Source, 18 février 2015. 
González-Barrera, Ana, 2015, « More Mexicans Leaving Than Coming to the U.S. », Pew Research Center, 19 novembre 2015.

Hernández, Kelly Lyle, Migra! A History of the U.S. Border Patrol, University of California Press, 2010.

Krogstad, Jens Manuel et Jeffrey S. Passsel, « 5 Facts about illegal immigration in the U.S. », Pew Research Center, 19 novembre 2015.

Massey, Douglas S., Jorge Durand et Nolan J. Malone, Beyond Smoke and Mirrors: Mexican Immigration in the Era of Economic Integration, Russell Sage Foundation, 2002.

Massey, Douglas S., "How a 1965 immigration reform created illegal immigration", Washington Post, 25 septembre 2015.

Massey, Douglas S. et Karen A. Pren, "Unintended Consequences of US Immigration Policy: Explaining the Post-1965 Surge from Latin America", Population Development Review no. 38, vol. 1 2012 (via NIH Public Access), p. 1-29.

Nevins, Joseph, Operation Gatekeeper: The Rise of the "Illegal Alien" and the Making of the U.S.-Mexico Boundary, Routledge, 2002.

Ngai, Mae M., Impossible Subjects: Illegal Aliens and the Making of Modern America, Princeton University Press, 2004.

Ong Hing, Bill, Defining America Through Immigration Policy, Temple University Press, 2003.

Reimer, David M., Unwelcome Strangers: American identity and the Turn Against Immigration, Columbia University Press, 1998.

Tichenor, Daniel J., Dividing Lines: The Politics of Immigration Control in America, Princeton University Press, 2002.

Zolberg, Aristide R., A Nation by Design: Immigration Policy in the Fashioning of America, Russell Sage Foundation/Harvard University Press, 2006.

\section{AUTEUR}

\section{JAMES COHEN}

James Cohen est professeur à l'Université de Paris 3 Sorbonne Nouvelle, CREW (EA 4399), auteur de À la poursuite des « illégaux ». Politiques et mouvements anti-immigrés aux États-Unis, Éditions Le Croquant, 2012. 